THU-96/39

quant-ph/9612018

\title{
QUANTUMMECHANICAL BEHAVIOUR IN A DETERMINISTIC MODEL
}

\author{
G. 't Hooft \\ Institute for Theoretical Physics \\ University of Utrecht, P.O.Box 80006 \\ 3508 TA Utrecht, the Netherlands \\ e-mail: g.thooft@fys.ruu.nl
}

\begin{abstract}
A deterministic model with a large number of continuous and discrete degrees of freedom is described, and a statistical treatment is proposed. The model exactly obeys a Schrödinger equation, which has to be interpreted exactly according to the Copenhagen prescriptions. After applying a Hartree-Fock approximation, the model appears to describe genuine quantum particles that could be used as a starting point for field variables in a quantum field theory. In the deterministic model it is essential that information loss occurs, but the corresponding quantum system is unitary and exactly preserves information.
\end{abstract}

This paper is intended to exhibit the mathematical nature of a model that could be used for further study of the quantum mechanical nature of this world, but the philosophical implications will not be addressed, nor will we discuss possible implications in connection with the usual quantummechanics paradoxes such as the Einstein Rosen Podolski paradox these are postponed to a more elaborate publication. In the present paper, the reader is invited to draw his or her own conclusions concerning the relevance of this model.

First, consider one continuous parameter $q(t) \in \mathbb{R}$ (boundary conditions on $q$ will be postponed to later) and one discrete parameter $s= \pm 1$, and let them obey the (deterministic) time evolution equations

$$
\frac{\mathrm{d}}{\mathrm{d} t} q(t)=s ; \quad \frac{\mathrm{d}}{\mathrm{d} t} s=0 .
$$


This means that we have a 'particle' moving along the real line with velocity either +1 or -1 . Defining the Hamiltonian

$$
H=s p
$$

allows us to write the Hamilton equations

$$
\frac{\mathrm{d}}{\mathrm{d} t} q=\{q, H\} ; \quad \frac{\mathrm{d}}{\mathrm{d} t} s=\{s, H\} ; \quad \text { if } \quad\{q, p\}=1, \quad\{s, p\}=\{s, q\}=0 .
$$

Of course, if we start with a probability distribution $P(q, s, 0)$ at $t=0$, we have as a solution

$$
P(q, s, t)=P(q-s t, s, 0),
$$

and one can write $P \equiv|\psi|^{2}$, where $\psi$ is a wave function, also satisfying

$$
\psi(q, s, t)=\psi(q-s t, s, 0) .
$$

This $\psi$ obeys the Schrödinger equation ${ }^{1}$

$$
\frac{\mathrm{d}}{\mathrm{d} t} \psi(q, s, t)=-i \hat{H} \psi(q, s, t), \quad \text { if } \quad \hat{H}=s \hat{p}, \quad \hat{p}=-i \frac{\partial}{\partial q}
$$

Writing things this way, we see that the 'quantum' model of Eq. 6 is physically and mathematically identical to the classical one. Here, we write 'quantum' between quotation marks because it can hardly be called a quantum theory in the usual sense: the wave function $\psi$ does not spread as time proceeds (so one cannot envisage interference experiments), and the Hamiltonian $\hat{H}$ is not bounded from below, so that there is no ground state. It is of importance, however, to emphasize that it is entirely legal to attach to this wave function $\psi$ a Copenhagen probability interpretation. Quantum mechanical superposition of states $\psi$ is permitted in the usual way. Later, we will see how a lower bound in the Hamiltonian of a true quantum theory may arise. A lower bound will be necessary since we wish to have a ground state, or vacuum state.

Next, let us take two sets of such variables, $q_{1}, q_{2}, s_{1}$ and $s_{2}$. Again, $q_{i}$ are real numbers and $s_{i}$ are \pm 1 . If the Hamiltonian is taken to be (from now on we omit the hats)

$$
H_{0}=s_{1} p_{1}+s_{2} p_{2}, \quad\left[q_{i}, p_{j}\right]=i \delta_{i j} ; \quad\left[s_{i}, s_{j}\right]=0
$$

then we have two (distinguishable) particles moving as before.

Now, we introduce as interaction

$$
s_{1} \rightarrow-s_{1} \quad \text { if } \quad q_{2}=c
$$


where $c$ is a fixed number. This means that particle 1 flips its velocity whenever particle 2 crosses the point $c$. Clearly, this is a deterministic law. We can express this interaction by means of an extra term in the quantum Hamiltonian:

$$
H=H_{0}+H_{1} ; \quad H_{1}=\frac{1}{2} \pi\left(\sigma_{1}^{1}-1\right) \delta\left(q_{2}-c\right)
$$

where we introduced the Pauli matrices

$$
\sigma_{i}^{3} \equiv s_{i}, \quad\left[\sigma_{i}^{a}, \sigma_{j}^{b}\right]=2 i \delta_{i j} \varepsilon^{a b c} \sigma_{i}^{c}
$$

This Hamiltonian works because $q_{2}$ moves with a fixed velocity; the factor $\frac{1}{2} \pi$ is exactly what is needed to flip $\sigma_{1}^{3}$ over. The -1 in Eq. 8 is there to ensure that the effect of this Hamiltonian is a flip without a sign change in the wave function:

$$
\exp \left(-\frac{1}{2} i \pi\left(\sigma_{1}^{1}-1\right)\right)=\sigma_{1}=\left(\begin{array}{ll}
0 & 1 \\
1 & 0
\end{array}\right)
$$

The positive signs here are important because we wish to find solutions for $\psi$ that are either positive real numbers or at most have slowly varying phase factors, not depending very much on $s_{i}$. This will allow us later to construct approximate solutions. The overall sign in the Hamiltonian $H_{1}$ is at first sight arbitrary, but this choice turns out to be important later (see remarks near the end of this paper).

Next, it will be of essential importance to introduce information loss at the deterministic level: states that are initially different may evolve into the same final state. This is what will make our model truly quantum mechanical. The resulting quantum theory will still be time-reversible and unitary, because the physical states will be defined to be only those that can be reached in the infinite future ${ }^{2}$. When $q_{2}$ passes a point $a$, the discrete variable $s_{1}$ will jump to +1 if it was -1 before, and if it was +1 it will stay +1 . This is described by the matrix

$$
\left(\begin{array}{ll}
1 & 1 \\
0 & 0
\end{array}\right)=\frac{1}{2}\left(i \sigma^{2}+\sigma^{3}+\sigma^{1}+1\right)
$$

Since

$$
\exp \left(i \alpha \sigma^{2}+\alpha\left(\sigma^{3}+\sigma^{1}-1\right)\right)=\left(\begin{array}{cc}
1 & 1-e^{-2 \alpha} \\
0 & e^{-2 \alpha}
\end{array}\right)
$$

we can use the interaction Hamiltonian

$$
H_{2}=\alpha\left(-\sigma^{2}+i\left(\sigma^{1}+\sigma^{3}-1\right)\right) \delta\left(q_{2}-a\right)
$$

where $\alpha$ is big, but not too big for our later approximation scheme. Note that $H_{2}$ by itself has one real Eigenvalue 0 with Eigenvector $|\psi(1)\rangle=\left(\begin{array}{l}1 \\ 0\end{array}\right)$ and a complex one, 
$-2 i \alpha \delta\left(q_{2}-a\right)$, with Eigenvector $|\psi(2)\rangle=\frac{1}{\sqrt{2}}\left(\begin{array}{c}1 \\ -1\end{array}\right)$. In the total Hamiltonian, at a later stage, we will keep only the real Eigenvalues. Similarly, the interaction Hamiltonian

$$
H_{3}=\beta\left(\sigma^{2}+i\left(\sigma^{1}-\sigma^{3}-1\right)\right) \delta\left(q_{2}-b\right)
$$

with sufficiently large $\beta$, will turn the spin $s_{1}$ to -1 if $q_{2}$ passes the point $b$. Again, additive constants in the Hamiltonians $H_{2}$ and $H_{3}$ have been chosen such that the transition matrices such as Eq. (11) carry positive signs only.

So-far, these Hamiltonians do not look useful for simulating more interesting quantum systems such as harmonic oscillators. But now we concentrate on the many-particle case.* Let there be $N$ continuous degrees of freedom $q_{i}, i=1, \ldots, N$, and $N$ discrete operators $s_{i}=\sigma_{i}^{3}$. Let $p_{i}$ and $\sigma_{i}^{a}$ obey the commutation rules (7) and (9). The Hamiltonian is generalized into

$$
\begin{aligned}
H= & \sum_{i} \sigma_{i}^{3} p_{i}+\sum_{i, j, k}\left(\frac{1}{2} \pi\left(\sigma_{i}^{1}-1\right) \delta\left(q_{j}-c_{i j}^{k}\right)+\alpha\left(-\sigma_{i}^{2}+i\left(\sigma_{i}^{1}+\sigma_{i}^{3}-1\right)\right) \delta\left(q_{j}-a_{i j}^{k}\right)+\right. \\
& \left.+\beta\left(\sigma_{i}^{2}+i\left(\sigma_{i}^{1}-\sigma_{i}^{3}-1\right)\right) \delta\left(q_{j}-b_{i j}^{k}\right)\right)
\end{aligned}
$$

The big summation here goes over values of $j \neq i$, and at each pair $(i, j)$ there may be several $k$ values.

The points $a_{i j}^{k}, b_{i j}^{k}$ and $c_{i j}^{k}$ are the free parameters of the model. As must be clear from the foregoing, this model is entirely deterministic; when $q_{j}$ takes one of the values $c_{i j}^{k}$, the velocity of the coordinate $q_{i}$ changes sign, if $q_{j}=a_{i j}^{k}$, this velocity becomes +1 , if $q_{j}=b_{i j}^{k}$, this velocity becomes -1 .

We are particularly interested in the case where, for any given $j$, the points $c_{i j}^{k}$ are fairly densely and smoothly distributed (the points $a_{i j}^{k}$ and $b_{i j}^{k}$, where information loss takes place, may be very scarce.) We imagine that the deterministic model will become chaotic. In order to approximate its behaviour statistically, we construct an approximate solution of the Eigenvalues and Eigenstates of $H$, using a 'Hartree-Fock' approximation. Take a trial wave function $\psi$ of the form

$$
\psi\left(\left\{q_{i}, s_{i}\right\}\right)=\prod_{i} \psi_{i}\left(q_{i}, s_{i}\right)
$$

where $\psi_{i}$ are sufficiently smooth single-particle wave functions.

\footnotetext{
* In a more realistic theory, at a later stage, we may be interested in regarding the $q_{i}$ as field values, and replace the index $i$ by a 3-space coordinate.
} 
Standard variation techniques tell us that the best functions $\psi_{i}$ are obtained as follows. Define

$$
\begin{gathered}
\frac{1}{2} \pi \sum_{j, k}\left\langle\psi_{j}\left|\delta\left(q_{j}-c_{i j}^{k}\right)\right| \psi_{j}\right\rangle=C_{i} \\
\alpha \sum_{j, k}\left\langle\psi_{j}\left|\delta\left(q_{j}-a_{i j}^{k}\right)\right| \psi_{j}\right\rangle=A_{i} \\
\beta \sum_{j, k}\left\langle\psi_{j}\left|\delta\left(q_{j}-b_{i j}^{k}\right)\right| \psi_{j}\right\rangle=B_{i} \\
\sum_{i, k}\left\langle\psi_{i}\right| \frac{1}{2} \pi \delta\left(q_{j}-c_{i j}^{k}\right)\left(\sigma_{i}^{1}-1\right)+\alpha\left(-\sigma_{i}^{2}+i\left(\sigma_{i}^{1}+\sigma_{i}^{3}-1\right)\right) \delta\left(q_{j}-a_{i j}^{k}\right)+ \\
+\beta\left(\sigma_{i}^{2}+i\left(\sigma_{i}^{1}-\sigma_{i}^{3}-1\right)\right) \delta\left(q_{j}-b_{i j}^{k}\right)\left|\psi_{i}\right\rangle=V_{j}\left(q_{j}\right)+i W_{j}\left(q_{j}\right) .
\end{gathered}
$$

The one-particle wave functions are then seen to obey

$$
\begin{gathered}
H_{i} \psi_{i}=E_{i} \psi_{i} ; \quad H_{i}=\sigma_{i}^{1}\left(C_{i}+i A_{i}+i B_{i}\right)+ \\
+\sigma_{i}^{2}\left(B_{i}-A_{i}\right)+\sigma_{i}^{3}\left(p_{i}+i A_{i}-i B_{i}\right)-C_{i}-i A_{i}-i B_{i}+V_{i}\left(q_{i}\right)+i W_{i}\left(q_{i}\right)
\end{gathered}
$$

If we are only interested in the Eigenvalues $E_{i}$, a renormalization of the wave functions allows us to replace $p_{i}$ by

$$
\hat{p}_{i} \equiv p_{i}+i A_{i}-i B_{i}
$$

The Eigenvalues $E_{i}$ are (for simplicity we drop the index $i$ ):

$$
\begin{aligned}
E & =-C-i(A+B)+V(q)+i W(q) \pm \sqrt{(C+i(A+B))^{2}+(B-A)^{2}+\hat{p}^{2}}= \\
& =-C-i(A+B)+V+i W \pm\left[C+i(A+B)+\frac{(B-A)^{2}+\hat{p}^{2}}{2(C+i(A+B))}+\mathcal{O}\left(\hat{p}^{4}\right)\right]
\end{aligned}
$$

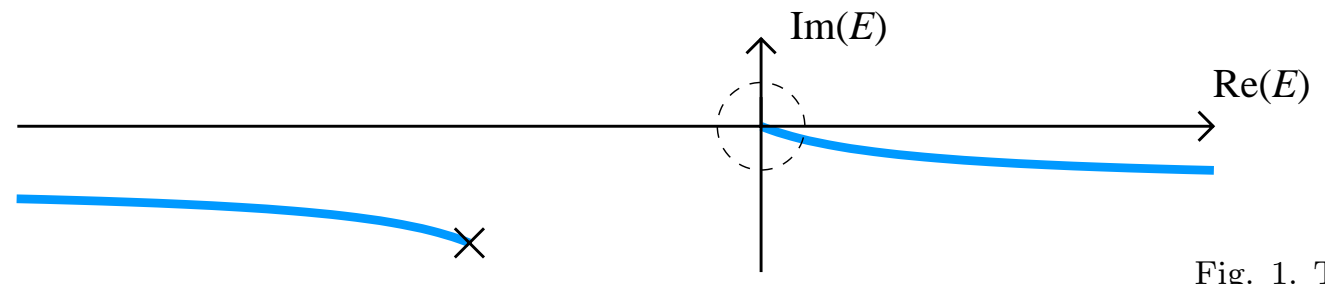

Fig. 1. The complex energy

Now, if we had no information loss, that is, $A=B=W=0$, we would have obtained a quantum particle with mass $\pm C$. The negative mass values are a serious problem here. In deterministic models, the negative Eigenvalues of the Hamiltonian have always been 
a serious difficulty. ${ }^{1,3}$ The most important result we wish to report here is the effect of information dissipation. We still assume $A$ and $B$ to be very small compared to $C$. In this case, the Eigenvalues with positive signs in Eq. (23) are practically real, whereas the others have substantial negative imaginary parts. These must be the states that do not survive at $t \rightarrow \infty$. The other states have a tiny negative imaginary part. Knowing that there must exist many real Eigenvalues, we imagine that higher order corrections will be big enough to remove the tiny imaginary parts for many of the lower energy states (inside the dotted circle in Figure 1.

We find that only the states with positive energies survive. This sign preference may be explained as follows. If we had chosen the signs in Eq. (10) oppositely, we would have found that only the negative energy values survive. Thus, the sign choice in Eq. (10) must be seen as establishing the sign convention for the entire Hamiltonian.

Corrections to the Hartree-Fock aproximation will give interactions between the particle-like objects. Since their matrix elements are non-vanishing, there will be contributions from virtual negative-energy states, but these will not occur in the final states because they are non-physical. By construction, the exact evolution matrix $U=e^{-i H t}$ will be unitary within the sector of the physical states, which is why we expect the exact physical spectrum to contain real $E$ values only.

Substituting the solutions for $\psi_{i}$ back into Eq. 20, we find that $V$ and $W$ vanish as soon as $B=A$, since then $\psi_{i}\left(q_{i}, s_{i}\right)=1$. But it is easy to introduce non-trivial boundary conditions for the $q$ 's, so that $\left\langle p_{i}^{2}\right\rangle \neq 0$, and $\left\langle\sigma_{i}^{1}\right\rangle \neq 0$, in which case non-trivial potentials $V$ and $W$ may emerge (with $|W| \ll|V|$ ). Here we observe the effects of the -1 in Eq. 8: it removes unnecessary large interaction terms so that the Hartree Fock approximation works optimally. There exist of course more ways to generalize the model. Most essential features seem te be the fact that there are continuous variables $q_{i}$ (so that an infinitesimal time shift can be defined), and discrete variables $s_{i}$ (allowing us to introduce information loss).

Our arguments claiming that non-Hartree Fock corrections will produce a real (and positive) energy spectrum are as yet delicate, and may be called inconclusive. We suggest that whether or not a non-trivial spectrum of real energy values emerges may depend on further details of the model. It will probably be essential that our model be chaotic, a feature that will depend on details in a complicated way. An other way to look at the model is to consider the $N \rightarrow \infty$ limit, and assume that every 'particle' interacts with every other one. ${ }^{\dagger}$ In this limit, $C$ may tend to infinity and if we keep $A$ and $B$ small, the

$\dagger$ See footnote on Page 4 
positive energy values will become real. We have a real quantum theory. Needless to say, the model can be generalized in many ways. Here, we merely wanted to point out some of its most essential features.

\section{References}

1. G. 't Hooft, J. Stat. Physics 53 (1988) 323; Nucl. Phys. B342 (1990) 471.

2. G. 't Hooft, to be publ.

3. G. 't Hooft, K. Isler and S. Kalitzin, Nucl. Phys. B386 (1992) 495. 